Pacific Journal of Mathematics

RELATIVE NIELSEN NUMBS 


\title{
A RELATIVE NIELSEN NUMBER
}

\author{
Helga SCHIRMER
}

\begin{abstract}
A relative Nielsen number $N(f ; X, A)$ for a selfmap $f:(X, A) \rightarrow$ $(X, A)$ of a pair of spaces is introduced which shares such properties with the Nielsen number $N(f)$ as homotopy invariance and homotopy type invariance. As $N(f ; X, A) \geq N(f)=N(f ; X, \varnothing)$, the relative Nielsen number is in the case $A \neq \varnothing$ a better lower bound than $N(f)$ for the minimum number $M F[f ; X, A]$ of fixed points of all maps in the homotopy class of $f$. Conditions for a compact polyhedral pair $(X, A)$ are given which ensure that the relative Nielsen number is in fact the best possible lower bound, i.e. that $N(f ; X, A)=M F[f ; X, A]$.
\end{abstract}

1. Introduction. Nielsen fixed point theory is concerned with the determination of the minimum number $M F[f]$ of fixed points in the homotopy class of a given map $f: X \rightarrow X$. For this purpose the so-called Nielsen number $N(f)$ is introduced, which is always a lower bound for $M F[f]$ and in many cases the best possible lower bound. (See e.g. [1] and [5] for background.) $N(f)$ can, however, be a very poor lower bound for $M F[f]$ if $f:(X, A) \rightarrow(X, A)$ is a selfmap of a pair of spaces and a homotopy a map of the form $H:(X \times I, A \times I) \rightarrow(X, A)$. To see this, consider the case where $X=B^{2}$ is a 2-disk and $A=S^{1}$ the circle bounding it. If $f:\left(B^{2}, S^{1}\right) \rightarrow\left(B^{2}, S^{1}\right)$ is a map which is of degree $d$ on $S^{1}$, then $f$ must have at least $|d-1|$ fixed points as even the restriction of $f$ to $S^{1}$ cannot have fewer fixed points ([1], Ch. VII C, p. 107, [5], p. 33, Example 1). But $N(f)=1$. Hence we need a "relative" Nielsen number for maps of pairs of spaces which is a better, and ideally sharp, lower bound for the minimum number $M F[f ; X, A]$ of fixed points in the homotopy class of the map $f:(X, A) \rightarrow(X, A)$.

It is the purpose of this paper to introduce such a relative Nielsen number $N(f ; X, A)$. The definition of $N(f ; X, A)$ in $\$ 2$ uses the existing definition of the fixed point classes of a selfmap of a single space. More precisely, $N(f ; X, A)$ is obtained by adding the number $N(f)$ of essential fixed point classes of $f: X \rightarrow X$ (i.e. of the map $f$ considered as a selfmap of $X$ only) and the number $N(\bar{f})$ of essential fixed point classes of the restriction $\bar{f}: A \rightarrow A$ of $f$ to $A$, and then subtracting the number $N(f, \bar{f})$ of "common" essential fixed point classes of $f$ and $\bar{f}$, where a common fixed point class of $f$ and $\bar{f}$ is defined as a fixed point class of $f$ which 
intersects an essential fixed point class of $\bar{f}$. (See Definition 2.1.) This definition of $N(f ; X, A)$ yields a positive integer which has the usual basic properties of the Nielsen number $N(f)$. It is homotopy invariant (Theorem 3.3) and homotopy type invariant (Theorem 3.5), and it is a lower bound for $M F[f ; X, A]$ which is always at least as good as $N(f)$ (Theorems 3.1 and 3.2). The computation of relative Nielsen numbers need not be harder than the computation of ordinary Nielsen numbers, and we show in some examples in $\S 2$ that it can be easy to find $N(f ; X, A)$ once $N(f)$ and $N(\bar{f})$ are known.

In the final three sections we consider the question whether $N(f ; X, A)$ is in fact the best possible lower bound for $M F[f ; X, A]$, i.e. whether there exists a map $g:(X, A) \rightarrow(X, A)$ homotopic to a given map $f$ : $(X, A) \rightarrow(X, A)$ which has precisely $N(f ; X, A)$ fixed points. The Minimum Theorem 6.2 shows that this is the case under fairly general assumptions on $(X, A)$. The construction of a map with a minimal fixed point set proceeds, as usual, in two steps. In the first one we homotope $f$ to a fix-finite map, but we take care to ensure that this map has only $N(\bar{f})$ fixed points on $A$ (Theorem 4.1). In the second one, which is carried out in $\S 6$, we unite fixed points in $X-A$ whenever possible.

The assumptions of the Minimum Theorem 6.2 must by necessity include those which are needed if $A=\varnothing$ or $A=X$, but this is not sufficient. The new asumption which arises in our situation is that $A$ can be "by-passed" in $X$ (Definition 5.1), which means that every path in $X$ which joins two points in $X-A$ can be homotoped away from $A$. Fortunately this condition is satisfied in interesting cases including the one where $A$ is the boundary of a manifold $M$, but it shows that the relative Nielsen number $N(f ; X, A)$ introduced here may not be the final solution of the problem of finding the least number of fixed points $M F[f ; X, A]$ for maps of pairs of spaces.

The material in this paper is not presented in a self-contained form, as two expositions of Nielsen theory are easily accessible in the books by $\mathrm{R}$. F. Brown [1] and Boju Jiang [5]. We assume that the reader is familiar with the corresponding results from these books concerning the Nielsen number $N(f)$ of a map $f: X \rightarrow X$, but refer to them frequently. As the Minimum Theorem 6.2 is a main goal of this paper, it is more convenient to emphasise the definition of $N(f)$ by F. Wecken [11] which uses paths between fixed points rather than the original one by J. Nielsen [7] which uses covering spaces. In the final section we also assume that the reader is familar with the proof of the minimum theorem for maps of Boju Jiang [2]. 
I want to thank the referee for his helpful suggestions.

2. The relative Nielsen number $N(f ; X, A)$. In this section we introduce the relative Nielsen number $N(f ; X, A)$ for maps of pairs of spaces $f:(X, A) \rightarrow(X, A)$, obtain some immediate consequences of the definition and illustrate it by some examples. The definition of $N(f ; X, A)$ uses the concepts of an essential fixed point class and of the Nielsen number for a compact (metric) ANR. They can e.g. be found in [1, Ch. VI, p. 85 ff.] and [5, Ch. I, p. 4 ff.].

If $(X, A)$ is a pair of spaces and $f:(X, A) \rightarrow(X, A)$ is a map, we shall write $\bar{f}: A \rightarrow A$ for the restriction of $f$ to $A$, and $f: X \rightarrow X$ for the map $f:(X, A) \rightarrow(X, A)$ if the condition that $f(A) \subset A$ is immaterial. Hence homotopies of $f:(X, A) \rightarrow(X, A)$ are maps of the form $H$ : $(X \times I, A \times I) \rightarrow(X, A)$ (where $I$ is the unit interval), and homotopies of $f: X \rightarrow X$ are maps of the form $H: X \times I \rightarrow X$. This abuse of the symbol $f$ is deliberate, and not likely to cause confusion. We write Fix $f$ for the fixed point set $\{x \in X \mid f(x)=x\}$ and $\mathbf{F}$ for a fixed point class of $f: X \rightarrow X$. The essential fixed point set Fix $f$ of $f: X \rightarrow X$ will be defined as

Fix $_{e} f=U\{x \in X \mid x$ lies in an essential fixed point class of $f\}$.

Similarly we write $\overline{\mathbf{F}}$ for a fixed point class of $\bar{f}: A \rightarrow A$, and $\operatorname{Fix}_{e} \bar{f}$ for its essential fixed point set.

Definition 2.1. Let $f:(X, A) \rightarrow(X, A)$ be a map of a pair of spaces. A fixed point class $\mathbf{F}$ of $f: X \rightarrow X$ is a common fixed point class of $f$ and $\bar{f}$ if $\mathbf{F} \cap \operatorname{Fix}_{e} \bar{f} \neq \varnothing$. It is an essential common fixed point class of $f$ and $\tilde{f}$ if it is an essential fixed point class of $f: X \rightarrow X$ and a common fixed point class of $f$ and $\bar{f}$.

An equivalent definition, which is useful in some later proofs, is contained in the corollary of the next lemma.

Lemma 2.2. Let $f:(X, A) \rightarrow(X, A)$ be a map, let $\mathbf{F}$ be a fixed point class of $f: X \rightarrow X$ and let $\overline{\mathbf{F}}$ be a fixed point class of $\bar{f}: A \rightarrow A$. If $\mathbf{F} \cap \overline{\mathbf{F}} \neq \varnothing$, then $\overline{\mathbf{F}} \subset \mathbf{F}$.

Proof. According to the definition of a fixed point class $x_{0}, x_{1} \in$ Fix $f$ belong to the same fixed point class $\mathbf{F}$ of $f: X \rightarrow X$ if there exists a path $p=\left\{x_{t}\right\}_{t \in I}$ in $X$ from $x_{0}$ to $x_{1}$ so that the paths $p$ and $f \circ p=$ $\left\{f\left(x_{t}\right)\right\}_{t \in I}$ are homotopic. (By a homotopy of paths we always mean one 
which keeps end points fixed.) Hence if $a_{0} \in \mathbf{F} \cap \overline{\mathbf{F}}$ and $a_{1} \in \overline{\mathbf{F}}$, then there exists a path $\left\{a_{t}\right\}_{t \in I}$ in $A$ from $a_{0}$ to $a_{1}$ so that $\left\{\bar{f}\left(a_{t}\right)\right\}_{t \in I}$ is homotopic to $\left\{a_{t}\right\}_{t \in I}$ in $A$. As $A \subset X$ this implies $a_{1} \in \mathbf{F}$.

Corollary 2.3. Let $f:(X, A) \rightarrow(X, A)$ be a map. A fixed point class F of $f: X \rightarrow X$ is a common fixed point class of $f$ and $\bar{f}$ if and only if it contains an essential fixed point class of $\bar{f}: A \rightarrow A$.

We write $N(f, \bar{f})$ for the number of essential common fixed point classes of $f$ and $\bar{f}$. If $X$ is a compact ANR, then $N(f, \bar{f})$ is finite as $0 \leq N(f, \bar{f}) \leq N(f)$.

Definition 2.4. Let $(X, A)$ be a pair of compact ANR's. If $f$ : $(X, A) \rightarrow(X, A)$ is a map, then its relative Nielsen number $N(f ; X, A)$ is defined as

$$
N(f ; X, A)=N(\bar{f})+N(f)-N(f, \bar{f}) .
$$

Hence $N(f ; X, A)$ is a finite integer $\geq 0$, and equals $N(f)$ if $X=A$ or $A=\varnothing$. The next two theorems list some other cases in which the relative Nielsen number equals an ordinary one.

THEOREM 2.5. Let $(X, A)$ be a pair of compact ANR's and let $f$ : $(X, A) \rightarrow(X, A)$ be a map.

(i) If $N(f)=0$, then $N(f ; X, A)=N(\bar{f})$,

(ii) if $N(\bar{f})=0$, then $N(f ; X, A)=N(f)$.

Proof. This is obvious from the definition, as in both cases $N(f, \bar{f})=$ 0 .

TheOREM 2.6. Let $(X, A)$ be a pair of compact ANR's and let $f$ : $(X, A) \rightarrow(X, A)$ be a map. If either $X$ is simply connected or if $X$ is connected and $f$ homotopic to the identity map id: $(X, A) \rightarrow(X, A)$, then

$$
N(f ; X, A)= \begin{cases}N(f) & \text { if } N(\bar{f})=0, \\ N(\bar{f}) & \text { if } N(\bar{f}) \neq 0 .\end{cases}
$$

Proof. We only have to consider the case where $N(f) \neq 0$ and $N(\bar{f}) \neq 0$. If $X$ is simply connected, then $f: X \rightarrow X$ has one essential fixed point class $\mathbf{F}$, and $\bar{f}: A \rightarrow A$ has at least one essential fixed point classs $\overline{\mathbf{F}}$. But if $x \in \mathbf{F}$ and $a \in \overline{\mathbf{F}}$, then $a$ is also a fixed point of $f: X \rightarrow X$ and is in the same fixed point class as $x$, so $N(f, \bar{f})=1$. If $X$ is 
connected and $f$ homotopic to id: $(X, A) \rightarrow(X, A)$, then the same argument applies.

We finish this section with three examples which show that the computation of $N(f ; X, A)$ can be easy once $N(f)$ and $N(\bar{f})$ are known.

EXAMPLE 2.7. Let $X=B^{n}$, where $n \geq 2$, be an $n$-dimensional ball and let $A$ consist of the boundary $(n-1)$-sphere of $B^{n}$ together with $k$ points in the interior of $B^{n}$. If id: $\left(B^{n}, A\right) \rightarrow\left(B^{n}, A\right)$ is the identity map, then

$$
N(\overline{\mathrm{id}})= \begin{cases}k & \text { if } n \text { is even, } \\ k+1 & \text { if } n \text { is odd, }\end{cases}
$$

and hence it follows from Theorem 2.6 that

$$
N\left(\mathrm{id} ; B^{n}, A\right)= \begin{cases}1 & \text { if } k=0 \\ k & \text { if } k \geq 1 \text { and } n \text { is even, } \\ k+1 & \text { if } k \geq 1 \text { and } n \text { is odd. }\end{cases}
$$

EXAMPLE 2.8. Let $X$ be the solid torus in Euclidean 3-space $R^{3}$ which is obtained by rotating the 2-disk in the $x_{1} x_{3}$-plane of radius 1 and centered at $(2,0,0)$ about the $x_{3}$-axis, and let $A$ be the 2-dimensional torus which bounds $X$. We consider $R^{3}$ as $\mathbf{C} \times R^{1}$, where $\mathbf{C}$ is the complex plane, and label the points of $X$ as $\left(r e^{i \theta}, t\right)$, where $r e^{i \theta} \in \mathbf{C}$ and $t \in R^{1}$, with $1 \leq r \leq 3,0 \leq \theta<2 \pi$ and $-1 \leq t \leq 1$. Let $f:(X, A) \rightarrow(X, A)$ be the map given by $f\left(r e^{i \theta}, t\right)=\left(r e^{i d \theta},-t\right)$, where $d \neq 1$ is an integer. As any circle of latitude is a deformation retract of $X$ we have $N(f)=|d-1|$ ([1], Ch. VIII, p. 107; [5], p. 21; Theorem 5.4 and p. 33, Example 1), and it follows from [5], p. 33, Example 2 that $N(\bar{f})=2|d-1|$. The fixed point set of $f$ lies in $t=0$ and consists of $|d-1|$ line segments. Each line segment forms an essential fixed point class of $f$ and contains two essential fixed point classes of $\bar{f}$ on its boundary. Hence $N(f, \bar{f})=N(f)$ $=|d-1|$, and $N(f ; X, A)=N(\bar{f})=2|d-1|$.

EXAmple 2.9. Let $X$ be a disk with two holes, let $T$ be the boundary of $X$ and $f$ the reflection on the axis $l$ (see Figure 1). Then $N(f)=1$, $N(\bar{f})=2$ and $N(f, \bar{f})=1$ imply $N(f ; X, A)=2$. As any homeomorphism of $X$ which is isotopic to $f$ maps $A$ onto $A$, it has at least 2 fixed points. (This is the example used by Boju Jiang [3, p. 169] to explain why in his realization theorem for the Nielsen number $N(h)$ of a surface homeomorphism $h$ (see [4], Main Theorem) it is necessary to allow isotopies through embeddings rather than through homeomorphisms if a boundary component of the surface is mapped onto itself in an orientation-reversing manner.) 


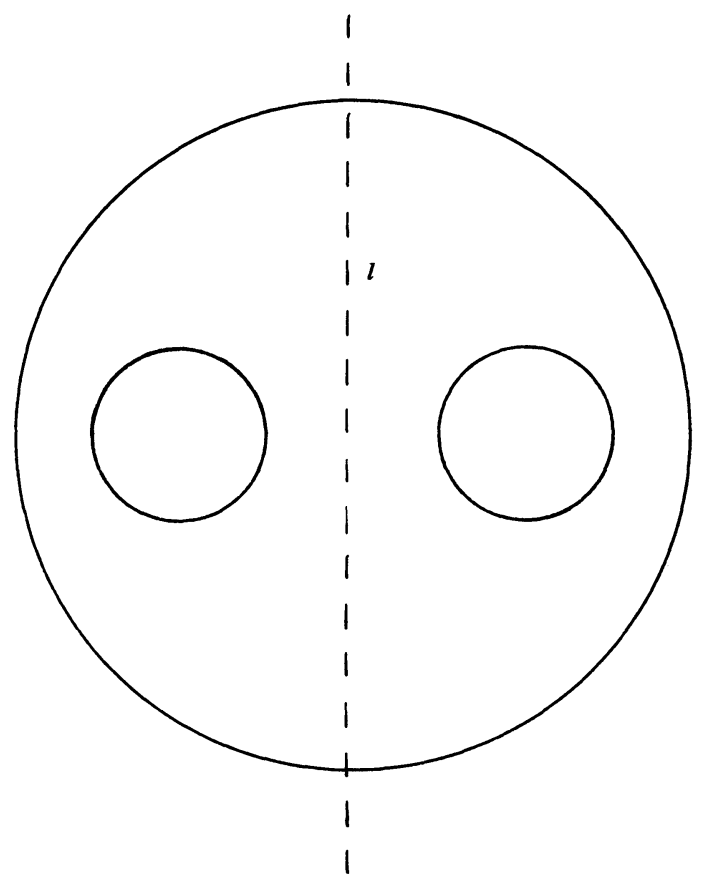

FIGURE 1

3. Basic properties of $N(f ; X, A)$. In this section we shall prove that $N(f ; X, A)$ is as lower bound for the number of fixed points of the map $f:(X, A) \rightarrow(X, A)$ (Theorem 3.1) and is usually sharper than $N(f)$ (Theorem 3.2). Then we show that $N(f ; X, A)$ is homotopy invariant (Theorem 3.3) and homotopy type invariant (Theorem 3.5).

THEOREM 3.1. (Lower bound property). If $(X, A)$ is a pair of compact $A N R$ 's, then every map $f:(X, A) \rightarrow(X, A)$ has at least $N(f ; X, A)$ fixed points.

Proof. Let the restriction $\bar{f}: A \rightarrow A$ of $f$ to $A$ have the essential fixed point classes $\overline{\mathbf{F}}_{1}, \overline{\mathbf{F}}_{2}, \ldots, \overline{\mathbf{F}}_{m}$, and let $f: X \rightarrow X$ have the essential fixed point classes $\mathbf{F}_{1}, \mathbf{F}_{2}, \ldots, \mathbf{F}_{n}, \mathbf{F}_{n+1}, \ldots, \mathbf{F}_{r}$ which are indexed so that the essential common fixed point classes of $f$ and $\bar{f}$ are $\mathbf{F}_{n+1}, \mathbf{F}_{n+2}, \ldots, \mathbf{F}_{r}$. Then

$$
N(f ; X, A)=m+r-(r-n)=m+n .
$$

Each fixed point class $\overline{\mathbf{F}}_{i}$ contains at least one fixed point $a_{i}$ of $\bar{f}$, and each fixed point class $\mathbf{F}_{J}$ contains at least one fixed point $x_{J}$ of $f$. If 
$j=1,2, \ldots, n$, then $\overline{\mathbf{F}}_{j} \cap A$ is distinct from $\operatorname{Fix}_{e} \bar{f}$, and so the set $\left\{a_{1}, a_{2}, \ldots, a_{m}, x_{1}, x_{2}, \ldots, x_{n}\right\}$ consists of $m+n$ distinct points which are all fixed points of $f:(X, A) \rightarrow(X, A)$.

THEOREM 3.2. If $(X, A)$ is a pair of compact ANR's and $f:(X, A) \rightarrow$ $(X, A)$ is a map, then $N(f ; X, A) \geq N(f)$ and $N(f ; X, A) \geq N(\bar{f})$.

Proof. As it follows from Corollary 2.3 that each essential common fixed point class of $f$ and $\bar{f}$ contains at least one essential fixed point class of $\bar{f}$, we have $N(f, \bar{f}) \leq N(\bar{f})$ and hence $N(f ; X, A)=N(f)+[N(\bar{f})-$ $N(f, \bar{f})] \geq N(f)$. From $N(f, \bar{f}) \leq N(f)$ follows $N(f ; X, A) \geq N(\bar{f})$.

THEOREM 3.3. (Homotopy invariance.) If $(X, A)$ is a pair of compact $A N R$ 's and if the maps $f_{0}, f_{1}:(X, A) \rightarrow(X, A)$ are homotopic, then $N\left(f_{0} ; X, A\right)=N\left(f_{1} ; X, A\right)$.

Proof. As it is well known that $N\left(f_{0}\right)=N\left(f_{1}\right)$ and $N\left(\bar{f}_{0}\right)=N\left(\tilde{f}_{1}\right)$ (see e.g. [1], Ch. VI E, Theorem 4, p. 95; [5], p. 19 Theorem 4.6), it suffices to show that $N(f, \bar{f})$ is invariant under homotopies $H:(X \times I, A \times I)$ $\rightarrow(X, A)$. So let $\mathbf{F}_{0}$ be an essential common fixed point class of $f_{0}$ and $\bar{f}_{0}$. Then $\mathbf{F}_{0}$ contains an essential fixed point class $\overline{\mathbf{F}}_{0}$ of $\bar{f}_{0}$ which is related under the restriction $\bar{H}$ of $H$ to $A \times I$ to an essential fixed point class $\overline{\mathbf{F}}_{1}$ of $\bar{f}_{1}$. This means that, for every $a_{0} \in \overline{\mathbf{F}}_{0}$ and $a_{1} \in \overline{\mathbf{F}}_{1}$, there exists a path $\left\{a_{t}\right\}_{t \in I}$ in $A$ from $a_{0}$ to $a_{1}$ so that the paths $\left\{\bar{h}_{t}\left(a_{t}\right)\right\}_{t \in I}$ and $\left\{a_{t}\right\}_{t \in I}$ are homotopic in $A$ (see [1], Ch. VI D and E, p. 87 ff., [5], Ch. I Theorem 2.9, p. 9).

Now let $\mathbf{F}_{1}$ be the fixed point class of $f_{1}$ which contains $a_{1}$. As $\left\{a_{t}\right\}_{t \in I}$ is a path in $X$ from $a_{0} \in \mathbf{F}_{0}$ to $a_{1} \in \mathbf{F}_{1}$ and as $\bar{h}_{t}\left(a_{t}\right)=h\left(a_{t}\right)$, the fixed point classes $\mathbf{F}_{0}$ of $f_{0}$ and $\mathbf{F}_{1}$ of $f_{1}$ are related under $H$. Hence $\mathbf{F}_{1}$ is an essential fixed point class of $f_{1}$, and as $F_{1} \cap \bar{F}_{1} \neq \varnothing$, it is a common fixed point class of $f_{1}$ and $\bar{f}_{1}$. Thus $H$ relates essential common fixed point classes of $f_{0}$ and $\bar{f}_{0}$ to essential common fixed point classes of $f_{1}$ and $\bar{f}_{1}$, and we have $N\left(f_{0}, \bar{f}_{0}\right)=N\left(f_{1}, \bar{f}_{1}\right)$.

The proofs of the next two theorems are quite analogous to those of the corresponding theorems for $N(f)$ which can be found in [5, Ch. I, Theorem 5.2, p. 20 and Theorem 5.4, p. 21]. Hence we leave the proofs to the reader. Two maps of pairs of spaces $f:(X, A) \rightarrow(X, A)$ and $g$ : $(Y, B) \rightarrow(Y, B)$ are said to be maps of the same homotopy type if there exists a homotopy equivalence $h:(X, A) \rightarrow(Y, B)$ so that the maps of pairs of spaces $h \circ f, g \circ h:(X, A) \rightarrow(Y, B)$ are homotopic. 
TheOREM 3.4. (Commutativity.) Let $(X, A)$ and $(Y, B)$ be two pairs of compact $A N R$ 's. If $f:(X, A) \rightarrow(Y, B)$ and $g:(Y, B) \rightarrow(X, A)$ are maps, then $N(g \circ f, \bar{g} \circ \bar{f})=N(f \circ g, \bar{f} \circ \bar{g})$ and hence

$$
N(g \circ f ; X, A)=N(f \circ g ; Y, B) .
$$

THEOREM 3.5. (Homotopy type invariance.) Let $(X, A)$ and $(Y, B)$ be two pairs of compact $A N R$ 's. If $f:(X, A) \rightarrow(X, A)$ and $g:(Y, B) \rightarrow(Y, B)$ are maps of the same homotopy type, then $N(f ; X, A)=N(g ; Y, B)$.

4. Fix-finite maps with minimal fixed point sets on a subspace. We have seen, in Theorems 3.1 and 3.3, that $N(f ; X, A)$ is a lower bound for $M F[f ; X, A]$. In the rest of this paper we want to show that, under suitable assumptions on $X$ and $A$, the relative Nielsen number $N(f ; X, A)$ is in fact a sharp lower bound for $M F[f ; X, A]$, i.e. that there exists a map $g:(X, A) \rightarrow(X, A)$ which is homotopic to $f:(X, A) \rightarrow(X, A)$ and has precisely $N(f ; X, A)$ fixed points. In the case where $A=\varnothing$ the construction of $g$ proceeds in two stages. In the first, $f: X \rightarrow X$ is homotoped to a fix-finite map (i.e. to a map with a finite fixed point set), and in the second fixed points in the same fixed point class are united (see [1], Ch. VIII, p. 115 ff., [2]). We now carry out similar constructions for a map of a pair of spaces $f:(X, A) \rightarrow(X, A)$. In this section we shall homotope $f$ to a fix-finite map $g:(X, A) \rightarrow(X, A)$, but already make sure that $g$ has only $N(\bar{f})$ fixed points on $A$ (Theorem 4.1).

As in the case $A=\varnothing$ we now have to restrict our attention to selfmaps of compact polyhedra, and we further have to assume that $A$ is a space for which $N(\bar{f})$ can be realized. We call a compact polyhedron $X$ a Nielsen space if every map $f: X \rightarrow X$ is homotopic to a map $g: X \rightarrow X$ which has $N(f)$ fixed points, and if these fixed points can lie anywhere in $X$. It is known that every compact connected polyhedron without a local cut point is a Nielsen space if it is not a surface of negative Euler characteristic [2, p. 760], and [8, Lemma 2, p. 525]. We write $\mathrm{Cl} Y$, Int $Y$ and $\mathrm{Bd} Y$ for the closure, interior and boundary in $X$ of a space $Y \subset X$.

THEOREM 4.1. Let $(X, A)$ be a pair of compact polyhedra, $X \neq A$, let $X$ be connected and let each component of $A$ be a Nielsen space. Then every map $f:(X, A) \rightarrow(X, A)$ is homotopic to a map $g:(X, A) \rightarrow(X, A)$ so that

(i) $\bar{g}$ has $N(\bar{f})$ fixed points which all lie on $\mathrm{Bd} A$,

(ii) $g$ is fix-finite,

(iii) all fixed points of $g$ on $X-A$ lie in maximal simplexes.

Proof. We shall proceed in two steps. 
Step 1. We show that $f:(X, A) \rightarrow(X, A)$ is homotopic to a map $h$ : $(X, A) \rightarrow(X, A)$ which has the following properties:

(a) $\bar{h}$ has $N(\bar{f})$ fixed points which all lie on $\operatorname{Bd} A$,

(b) there exists a compact polyhedron $B$ in $X$ so that $A \subset X-B$ and $h$ is fixed point free on $\mathrm{Cl}(X-B)-A$.

To construct $h$, we first use the fact that each component of $A$ is a Nielsen space in order to homotope $\bar{f}: A \rightarrow A$ to a selfmap of $A$ which has $N(\bar{f})$ fixed points which lie on $\operatorname{Bd} A$. Let $\bar{h}: A \rightarrow A$ be the resulting map and $\bar{F}: A \times I \rightarrow A$ be a homotopy from $\bar{f}$ to $\bar{h}$. The subpolyhedron $A$ is a strong deformation retract of some neighbourhood $V$ of $A$ in $X$. (See e.g. [10] Ch. 3.3, Cor. 11, p. 124.) Using a star covering of $A$ with respect to a subdivision of the triangulation of $X$ we can find a compact polyhedron $A_{1}$ with $A \subset \operatorname{Int} A_{1} \subset A_{1} \subset V$ so that $B=X-\operatorname{Int} A_{1}$ is a polyhedron in $X$. Let $R: A_{1} \times I \rightarrow V$ be the restriction to $A_{1}$ of the strong deformation retraction of $V$ onto $A$ and let $r: A_{1} \rightarrow A$ be the retraction given by $r(x)=R(x, 1)$. Then we can define a homotopy $F_{1}$ : $A_{1} \times I \rightarrow X$ by

$$
F_{1}(x, t)= \begin{cases}f \circ R(x, 2 t) & \text { if } 0 \leq t \leq \frac{1}{2}, \\ \bar{F}(r(x), 2 t-1) & \text { if } \frac{1}{2} \leq t \leq 1,\end{cases}
$$

and use the homotopy extension property of a polyhedral pair to extend $F_{1}$ to a homotopy $F:(X \times I, A \times I) \rightarrow(X, A)$ of $f$. If we define $h:(X, A) \rightarrow(X, A)$ by $h(x)=F(x, 1)$, then $h$ is fixed point free on $\mathrm{Cl}(X-B)-A \subset A_{1}-A$.

Step 2. We now show that $h:(X, A) \rightarrow(X, A)$ is homotopic to a map $g:(X, A) \rightarrow(X, A)$ which satisfies Theorem 4.1.

To do so, we write $d$ for the barycentric metric of $B$ and put $U=X-B$. As $h$ is fixed point free on $(\mathrm{Cl} U)-A$, there exists a $\delta>0$ so that $d(x, h(x))>\delta$ for all $x \in \operatorname{Bd} U$. With the help of the Hopf construction [1], Ch. VIII A, pp. 117-119 we change the restriction $h_{B}$ : $B \rightarrow X$ of $h$ to $B$ to a map $g_{B}: B \rightarrow X$ which is fix-finite, has all fixed points contained in maximal simplexes and is $\delta$-homotopic ([1], Ch. III A, p. 40) to $h_{B}$. Let $G_{B}: B \times I \rightarrow X$ be such a $\delta$-homotopy from $h_{B}$ to $g_{B}$. Then $G_{B}(x, t) \neq x$ for all $x \in \operatorname{Bd} U$.

If $G^{\prime}:\{((\mathrm{Cl} U) \times 0) \cup((\mathrm{Bd} U) \times I) \cup(A \times I), A \times I\} \rightarrow(X, A)$ is given by

$$
G^{\prime}(x, t)= \begin{cases}h(x) & \text { if }(x, t) \in((\mathrm{Cl} U) \times 0) \cup(A \times I), \\ G_{B}(x, t) & \text { if }(x, t) \in(\mathrm{Bd} U) \times I,\end{cases}
$$


then the restriction of $G^{\prime}$ to $((\operatorname{Bd} U) \cup A) \times I$ is a special homotopy [2, p. 751], and hence extends to a special homotopy $G_{U}:((\mathrm{Cl} U) \times I, A \times I)$ $\rightarrow(X, A)[2$, Lemma 2.1, p. 751]. We define a homotopy $G:(X \times I$, $A \times I) \rightarrow(X, A)$ by

$$
G(x, t)= \begin{cases}G_{U}(x, t) & \text { if }(x, t) \in(\mathrm{Cl} U) \times I, \\ G_{B}(x, t) & \text { if }(x, t) \in B \times I\end{cases}
$$

and a map $g:(X, A) \rightarrow(X, A)$ by $g(x)=G(x, 1)$. Then $g$ satisfies Theorem 4.1.

5. By-passing of subspaces. The Minimum Theorem 6.2 will be proved by uniting fixed points of the map $g$ constructed in Theorem 4.1 whenever possible. It will clearly be necessary to assume that $X$ and $A$ are triangulable Nielsen spaces, but we shall need one additional property of $(X, A)$ in order to realize $N(f ; X, A)$. This property is introduced in the next definition.

Definition 5.1. A subspace $A$ of a space $X$ can be by-passed if every path in $X$ with end points in $X-A$ is homotopic to a path in $X-A$.

Definition 5.1 states the property in the form in which it will be used in the proof of Theorem 6.2, but the next theorem states it in a form which is usually easier to verify. We write $i_{*}: \pi_{1}(X-A) \rightarrow \pi_{1}(X)$ for the homomorphism of the fundamental groups induced by the inclusion map. The easy proof of Theorem 5.2 is left to the reader.

TheOREM 5.2. Let $(X, A)$ be a pair of spaces and let $X$ be path-connected. Then $A$ can be by-passed in $X$ if and only if $X-A$ is path-connected and $i_{*}: \pi_{1}(X-A) \rightarrow \pi_{1}(X)$ is onto.

Examples of spaces which can be by-passed include the boundary Bd $M$ or a subpolyhedron $A$ of dimension $\operatorname{dim} A \leq \operatorname{dim} M-2$ of a triangulable manifold $M$. In particular, the subspaces $A$ in Examples 2.7-2.9 can be by-passed.

The next lemma will be used in the proof of Theorem 6.2. It contains the concept of a normal PL arc in a polyhedron $X=|K|$. An $\operatorname{arc} Q=q(I)$, where $q: I \rightarrow|K|$, is called in [2, p. 752], a normal PL arc if $q$ maps, for some subdivision $I^{\prime}$ of $I$, each simplex of $I^{\prime}$ linearly into a simplex of $|K|$, if it does not pass through any vertex of $|K|$ and if $q(s)$ lies in a maximal simplex of $|K|$ for all but a finite number of values $s \in I$ and goes from 
one maximal simplex into another when $s$ passes across any of these exceptional values. In our setting we have enlarged this definition. If $A$ is a subpolyhedron of $X=|K|$, we say that $Q$ is a normal $P L$ arc in $(|K|, A)$ if either $Q$ is a normal PL arc in $|K|-A$ or if $Q \cap A=\{q(1)\}$ and $Q$ is a normal PL arc in $|K|$ apart from the fact that $q(1)$ can be an arbitrary point of $\mathrm{Bd} A$.

LEMMA 5.3. Let $X=|K|$ be a compact connected polyhedron, let $A$ be a subpolyhedron so that $X-A$ has no local cut point and that $A$ can be by-passed, and let $p$ be a path in $|K|$ from a point $x_{0}$ in a maximal simplex of $|K|-A$ to a point $x_{1}$.

(i) If $x_{1} \neq x_{0}$ lies in a maximal simplex of $|K|-A$, then $p$ is homotopic to $q$ so that $q(I)$ is a normal PL arc in $(|K|, A)$ and $q(I) \cap A=$ $\varnothing$;

(ii) if $x_{1} \in \mathrm{Bd} A$, then $p$ is homotopic to $q$ such that $q(I)$ is a normal $P L$ arc in $(|K|, A)$ and $q(I) \cap A=\left\{x_{1}\right\}$.

Proof. (i) follows immediately from Definition 5.1 and [2], Lemma 3.3. If, as in (ii), $x_{1} \in \mathrm{Bd} A$, then we pick $y \in|K|-A$ near $x_{1}$ so that there exists a path $v: I \rightarrow|K|$ from $x_{1}$ to $y$ with $v(I) \cap A=\left\{x_{1}\right\}$ (see Figure 2). Then the composite path $p * v$ from $x_{0}$ to $y$ is homotopic to a path $p^{\prime}$ in $|K|-A$, and the desired path $q$ can be obtained from $p^{\prime} * v^{-1}$ along the lines of the proof of [2, Lemma 3.3], where only one "loose end" is used.

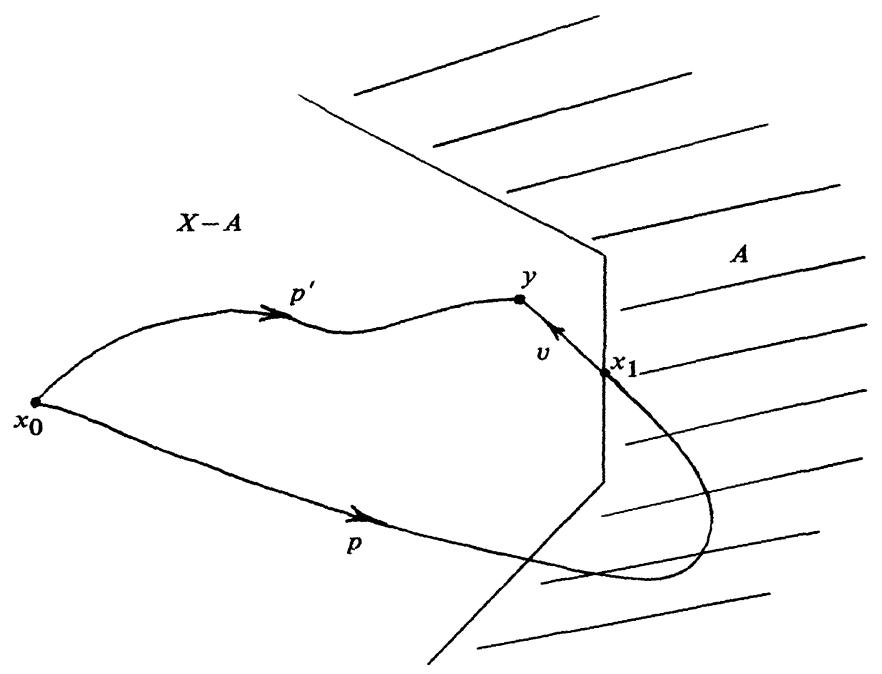

Figure 2 
6. The Minimum Theorem. The proof of the Minimum Theorem 6.2 is based on the proof of the corresponding theorem for the case $A=\varnothing$ by Boju Jiang [2, Theorem 5.2]. The assumptions on $(X, A)$ in Theorem 6.2 cannot be relaxed, as it is known that the Minimum Theorem is false if $A=\varnothing$ and $X$ is a surface [6] or a polyhedron with a local cut point [9].

We extract a part of the proof of Theorem 6.2 as Lemma 6.1, which is the form needed here of the fact that two fixed points $x_{0}$ and $x_{1}$ of a map $f: X \rightarrow X$ can be united if the restriction of $f$ to an arc $Q$ from $x_{0}$ to $x_{1}$ is homotopic to a proximity map [2, Lemma 2.2]. Due to Theorem 4.1 and Lemma 5.3 we shall only have to unite fixed points which satisfy the assumptions of the next lemma.

LemMA 6.1. Let $(X, A)=(|K|,|L|)$ be a pair of compact polyhedra, where $X$ is connected and $X-A$ has no local cut point and is not a 2-manifold. Let $x_{0}$ and $x_{1}$ be two isolated fixed points of a map $f$ : $(X, A) \rightarrow(X, A)$, and let $Q$ be a normal $P L$ arc in $(|K|, A)$ from $x_{0}$ to $x_{1}$, with Fix $f \cap Q=\left\{x_{0}, x_{1}\right\}$. Suppose that $x_{0}$ lies in a maximal simplex of $|K|-A$, and that $x_{1}$ lies either in a maximal simplex of $|K|-A$ or on $\mathrm{Bd} A$. Then there exists an $\varepsilon>0$ so that if $f \mid Q$ is specially homotopic to a map $g: Q \rightarrow X$ with $d(x, g(x))<\varepsilon$ for all $x \in Q$, then $f$ is homotopic to a map $f^{\prime}:(X, A) \rightarrow(X, A)$ with Fix $f^{\prime}=$ Fix $f-\left\{x_{0}\right\}$.

Proof. Let $Q=q(I)$, where $q: I \rightarrow K$, and let $K^{\prime}$ be the first barycentric subdivision of $K$. Shifting the new vertices if necessary, we can assume that $Q, x_{0}, x_{1}$ still satisfy the assumptions of Lemma 6.1 with respect to $K^{\prime}$. We select $\varepsilon>0$ so that $d(x, g(x))<\varepsilon$ for all $x \in Q$ implies that $g: Q \rightarrow X$ is a proximity map with respect to $K^{\prime}[\mathbf{1}, \mathrm{Ch}$. VIII C, p. 124], [2, p. 751].

By assumption there exists a special homotopy $G_{Q}: Q \times I \rightarrow X$ from $f \mid Q$ to $g: Q \rightarrow X$. We extend it to a special homotopy $g:\{(Q \cup A) \times I$, $A \times I\} \rightarrow(X, A)$ by putting

$$
G(x, t)= \begin{cases}G_{Q}(x, t) & \text { if }(x, t) \in Q \times I, \\ \bar{f}(x) & \text { if }(x, t) \in A \times I,\end{cases}
$$

and then use [2, Lemma 2.1] (with $A \cup Q$ instead of $A$ ) to extend $G$ to a special homotopy $H:(X \times I, A \times I) \rightarrow(X, A)$ which starts at $H(x, 0)=$ $f(x)$. If $f^{\prime \prime}:(X, A) \rightarrow(X, A)$ is given by $f^{\prime \prime}(x)=H(x, 1)$, then $f^{\prime \prime}(x)=$ $g(x)$ for $x \in Q$. 
As $g: Q \rightarrow X$ is a proximity map with respect to $K^{\prime}$, we can move the fixed point $x_{0}$ of $f^{\prime \prime}$ along $Q$ to a point $x_{2}$ which lies in a maximal simplex $\left|\sigma^{\prime}\right|$ of $\left|K^{\prime}\right|$ with $x_{1} \in\left|\bar{\sigma}^{\prime}\right|$ in such a way that the restriction of the map to $Q$ remains a proximity map with respect to $K^{\prime}[1, \mathrm{Ch}$. VIII C, Lemma 3, p. 128]. If $Q \subset X-A$, we can then unite $x_{2}$ with $x_{1}$ as usual $\left[1\right.$, Ch. VIII C, Lemma 2, p. 126] to obtain a map $f^{\prime}:(X, A) \rightarrow(X, A)$ with Fix $f^{\prime}=$ Fix $f-\left\{x_{0}\right\}$.

But we still have to unite the fixed points $x_{2}$ and $x_{1}$ in the case $Q \cap A=\left\{x_{1}\right\}$. So let $f_{1}:(X, A) \rightarrow(X, A)$ be a map which is homotopic to $f:(X, A) \rightarrow(X, A)$ and has $x_{2} \in\left|\sigma^{\prime}\right|$ and $x_{1} \in\left|\bar{\sigma}^{\prime}\right| \cap A$ as isolated fixed points, where $\left|\sigma^{\prime}\right|$ is a maximal simplex in $\left|K^{\prime}\right|-A$, and let the restriction of $f_{1}$ to the half-open segment $\left[x_{2}, x_{1}\right)$ in $\left|\sigma^{\prime}\right|$ be a proximity map with respect to $K^{\prime}$. Let $U$ be an open neighbourhood of $\left[x_{2}, x_{1}\right)$ in $|\sigma|$ with $(\operatorname{Bd} U) \cap A=\left\{x_{1}\right\}$ and $f_{1}(\mathrm{Cl} U) \subset W$, and so that $\mathrm{Cl} U$ is convex with respect to the simplicial structure of $|K|$ (see Figure 3). The points of $(\mathrm{Cl} U)-\left\{x_{1}\right\}$ can be labelled as $x=\left(b_{x}, t_{x}\right)$, where $b_{x} \in \mathrm{Bd} U$, $0<t_{x} \leq 1$ and

$$
x=t_{x} b_{x}+\left(1-t_{x}\right) x_{1}
$$

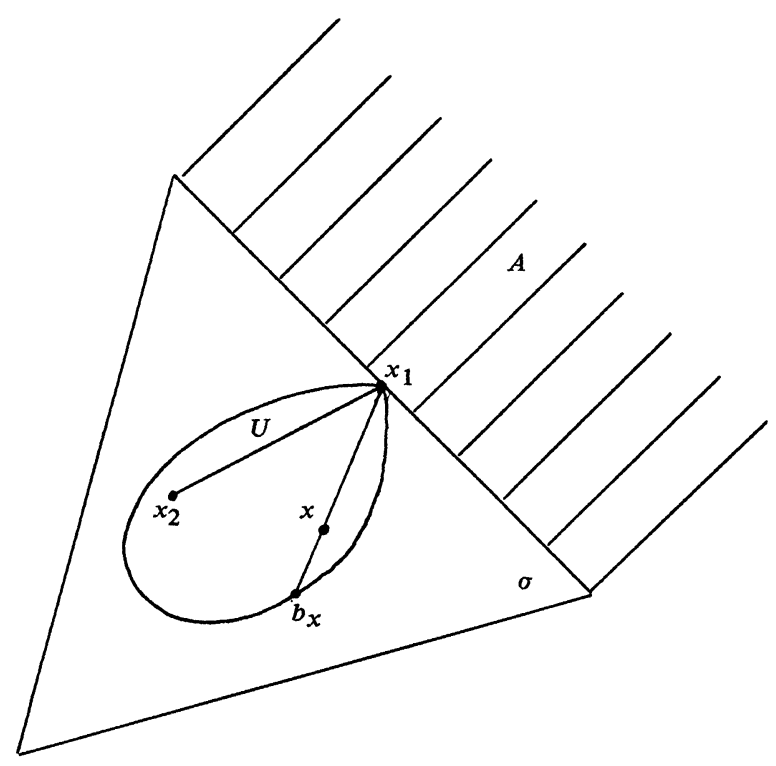

Figure 3 
with respect to the simplicial structure of $|K|$. Let $f^{\prime}:(X, A) \rightarrow(X, A)$ be defined by

$$
f^{\prime}(x)= \begin{cases}x_{1} & \text { if } x=x_{1} \\ t_{x} f_{1}\left(b_{x}\right)+\left(1-t_{x}\right) x_{1} & \text { if } x=\left(b_{x}, t_{x}\right) \in(\mathrm{Cl} U)-\left\{x_{1}\right\} \\ f_{1}(x) & \text { if } x \in X-\mathrm{Cl} U\end{cases}
$$

Then $f^{\prime}$ is a map homotopic to $f:(X, A) \rightarrow(X, A)$ and Fix $f^{\prime}=$ Fix $f-$ $\left\{x_{0}\right\}$.

We now prove a minimum theorem for maps of pairs of spaces. Note that its assumptions are satisfied in the Examples 2.7 (with $n \geq 3$ ) and 2.8 , hence the relative Nielsen numbers $N(f ; X, A)$ calculated in these examples can be realized.

Theorem 6.2. (Minimum Theorem). Let $(X, A)$ be a pair of compact polyhedra so that

(i) $X$ is connected,

(ii) $X-A$ has no local cut point and is not a 2-manifold,

(iii) every component of $A$ is a Nielsen space,

(iv) $A$ can be by-passed.

Then any map $f:(X, A) \rightarrow(X, A)$ is homotopic to a map $g:(X, A) \rightarrow$ $(X, A)$ with $N(f ; X, A)$ fixed points.

Proof. We can assume that $X \neq A$, as otherwise Theorem 6.2 is known. Let $(K, L)$ be a triangulation of $(X, A)$. Due to Theorem 4.1 we can assume that $\bar{f}$ has $N(\bar{f})$ fixed points which all lie on $\mathrm{Bd} A$, that $f$ is fix-finite on $X-A$ and that all fixed points of $f$ on $X-A$ lie in maximal simplexes of $|K|$. We now unite fixed points belonging to the same fixed point class $\mathbf{F}$ of $f: X \rightarrow X$. It suffices to show:

(6.3) Assume that either $\mathbf{F}$ is a common fixed point class of $f$ and $\bar{f}$ and $x_{0} \in \mathbf{F} \cap(X-A)$ or that $\mathbf{F}$ is not a common fixed point class of $f$ and $\bar{f}$ (and hence $\mathbf{F} \cap A=\varnothing$ ) and $x_{0}, x_{1} \in \mathbf{F}$. Then $f$ is homotopic to a $\operatorname{map} f^{\prime}:(X, A) \rightarrow(X, A)$ with Fix $f^{\prime}=$ Fix $f-\left\{x_{0}\right\}$.

Repetition of (6.3) and deletion of non-common fixed point classes which consist of a single fixed point of index zero by the usual method [1, Ch. VIII B, Theorem 4, p. 123] will lead to a map $g:(X, A) \rightarrow(X, A)$ with $N(f ; X, A)$ fixed points.

So let $\mathbf{F}$ be a fixed point class of $f: X \rightarrow X$ and $x_{0} \in \mathbf{F} \cap(X-A)$. If $\mathbf{F}$ is a common fixed point class of $f$ and $\bar{f}$, we can select $x_{1} \in \mathbf{F} \cap \operatorname{Bd} A$, 
if $\mathbf{F}$ is not a common fixed point class of $f$ and $\bar{f}$, we let $x_{1}$ be any point in $\mathbf{F}-\left\{x_{0}\right\}$. According to Lemma 5.3 there exists a path $q: I \rightarrow X$ from $x_{0}$ to $x_{1}$ which is homotopic to $f \circ q$ and has the property that $Q=q(I)$ is a normal PL arc in $(|K|, A)$ with $Q \cap A=\varnothing$ if $x_{1} \in X-A$ and $Q \cap A=\left\{x_{1}\right\}$ if $x_{1} \in \operatorname{Bd} A$. As all modifications from $p$ to $q$ in the proof of [2, Lemma 3.4] take place away from the end points, we can also ensure that $q$ satisfies the conditions $(\alpha)$ and $(\beta)$ of [2, Lemma 3.4], where $\tau$ and $\sigma_{1}$ are chosen in $X-A$. With the help of this $\operatorname{arc} q$ the proof of (6.3) can now be completed along the lines of the proof of [2, Theorem 5.2]. We choose $\varepsilon>0$ so that Lemma 6.1 applies and define a path $p_{\varepsilon}$ : $I \rightarrow X$ which is homotopic to $q$ by

$$
p_{\varepsilon}(s)=q(s+\delta \sin s \pi),
$$

where $\delta=\delta(\varepsilon)>0$ is determined so that $d\left(p_{\varepsilon}(s), q(s)\right)<\varepsilon$ for all $s \in I$. Then $p_{\varepsilon}$ and $f \circ q$ are special paths with respect to $Q[2, \mathrm{p}$. 755], and are homotopic. As in the proof of [2, Lemma 5.1] it follows that $p_{\varepsilon}$ and $f \circ q$ are specially homotopic. Hence the maps $f \mid Q: Q \rightarrow X$ and $p_{\varepsilon} \circ q^{-1}$ : $Q \rightarrow X$ are specially homotopic [2, p. 755], and therefore Lemma 6.1 (with $p_{\varepsilon} \circ q^{-1}$ instead of $g$ ) shows that (6.3) is true.

\section{REFERENCES}

[1] R. F. Brown, The Lefschetz Fixed Point Theorem, Scott, Foresman and Co., Glenview, Ill., 1971.

[2] Boju Jiang, On the least number of fixed points, Amer. J. Math., 102 (1980), 749-763.

[3] _ Fixed point classes from a differentiable viewpoint, Fixed Point Theory (Proceedings, Sherbrooke, Quebec, 1980), Springer-Verlag, Berlin, 1981. (Lecture Notes in Mathematics v. 886.)

[4] Fixed points of surface homeomorphisms, Bull. Amer. Math. Soc., 5 (1981), 176-178.

[5] Lectures on Nielsen Fixed Point Theory, Contemporary Mathematics v. 14, Amer. Math. Society, Providence, Rhode Island, 1983.

[6] _ Fixed points and braids, Invent. Math., 75 (1984), 69-74.

[7] J. Nielsen, Untersuchungen zur Topologie der geschlossenen zweiseitigen Flächen I, Acta Math., 50 (1927), 189-358.

[8] H. Schirmer, Mappings of polyhedra with prescribed fixed points and fixed point indices, Pacific J. Math., 63 (1976), 521-530.

[9] G.-H. Shi, Least number of fixed points of the identity class, Acta Math. Sinica, 18 (1975), 192-202.

[10] E. H. Spanier, Algebraic Topology, McGraw-Hill, New York, 1966.

[11] F. Wecken, Fixpunktklassen III, Math. Ann., 118 (1942), 544-577.

Received December 3, 1984 and in revised form April 25, 1985. This research was partially supported by NSERC Grant A 7579. 



\section{PACIFIC JOURNAL OF MATHEMATICS EDITORS}

\author{
V. S. VARAdarajan (Managing Editor) \\ University of California \\ Los Angeles, CA 90024 \\ Hebert Clemens \\ University of Utah \\ Salt Lake City, UT 84112 \\ Charles R. DePrima \\ California Institute of Technology \\ Pasadena, CA 91125
}

R. FINN

Stanford University

Stanford, CA 94305

HeRManN FLASChKa

University of Arizona

Tucson, AZ 85721

RAMESH A. GANGOlli

University of Washington

Seattle, WA 98195

ROBION KIRBY

University of California

Berkeley, CA 94720

\author{
C. C. MOORE \\ University of California \\ Berkeley, CA 94720 \\ H. SAMELSON \\ Stanford University \\ Stanford, CA 94305 \\ HAROLD STARK \\ University of California, San Diego \\ La Jolla, CA 92093
}

\section{ASSOCIATE EDITORS}

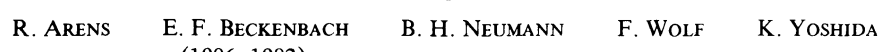

(1906-1982)

\section{SUPPORTING INSTITUTIONS}

\begin{abstract}
UNIVERSITY OF ARIZONA
UNIVERSITY OF BRITISH COLUMBIA

CALIFORNIA INSTITUTE OF TECHNOLOGY

UNIVERSITY OF CALIFORNIA

MONTANA STATE UNIVERSITY

UNIVERSITY OF NEVADA, RENO

NEW MEXICO STATE UNIVERSITY

OREGON STATE UNIVERSITY
\end{abstract}

\author{
UNIVERSITY OF OREGON \\ UNIVERSITY OF SOUTHERN CALIFORNIA \\ STANFORD UNIVERSITY \\ UNIVERSITY OF HAWAII \\ UNIVERSITY OF TOKYO \\ UNIVERSITY OF UTAH \\ WASHINGTON STATE UNIVERSITY \\ UNIVERSITY OF WASHINGTON
}

The Supporting Institutions listed above contribute to the cost of publication of this Journal, but they are not owners or publishers and have no responsibility for its content or policies.

Mathematical papers intended for publication in the Pacific Journal of Mathematics should be in typed form or offset-reproduced (not dittoed), double spaced with large margins. Please do not use built up fractions in the text of the manuscript. However, you may use them in the displayed equations. Underline Greek letters in red, German in green, and script in blue. The first paragraph must be capable of being used separately as a synopsis of the entire paper. In particular it should contain no bibliographic references. Please propose a heading for the odd numbered pages of less than 35 characters. Manuscripts, in triplicate, may be sent to any one of the editors. Please classify according to the scheme of Math. Reviews, Index to Vol. 39. Supply name and address of author to whom proofs should be sent. All other communications should be addressed to the managing editor, or Elaine Barth, University of California, Los Angeles, California 90024.

There are page-charges associated with articles appearing in the Pacific Journal of Mathematics. These charges are expected to be paid by the author's University, Government Agency or Company. If the author or authors do not have access to such Institutional support these charges are waived. Single authors will receive 50 free reprints; joint authors will receive a total of 100 free reprints. Additional copies may be obtained at cost in multiples of 50 .

The Pacific Journal of Mathematics is issued monthly as of January 1966. Regular subscription rate: $\$ 190.00$ a year (5 Vols., 10 issues). Special rate: $\$ 95.00$ a year to individual members of supporting institutions.

Subscriptions, orders for numbers issued in the last three calendar years, and changes of address should be sent to Pacific Journal of Mathematics, P.O. Box 969, Carmel Valley, CA 93924, U.S.A. Old back numbers obtainable from Kraus Periodicals Co., Route 100, Millwood, NY 10546.

The Pacific Journal of Mathematics at P.O. Box 969, Carmel Valley, CA 93924 (ISSN 0030-8730) publishes 5 volumes per year. Application to mail at Second-class postage rates is pending at Carmel Valley, California, and additional mailing offices. Postmaster: Send address changes to Pacific Journal of Mathematics, P.O. Box 969, Carmel Valley, CA 93924.

PUBLISHED BY PACIFIC JOURNAL OF MATHEMATICS, A NON-PROFIT CORPORATION

Copyright $\odot 1986$ by Pacific Journal of Mathematics 


\section{Pacific Journal of Mathematics}

\section{Vol. 122, No. 2 \\ February, 1986}

Gideon Amit and David Chillag, On a question of Feit concerning character values of finite solvable groups ......................257

Constantin Gelu Apostol and Frank Larkin Gilfeather, Isomorphisms modulo the compact operators of nest algebras ................263

Parviz Azimi and James Neil Hagler, Examples of hereditarily $l^{1}$ Banach

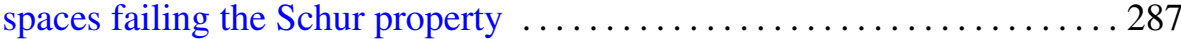

Brian Evan Blank, Boundary behavior of limits of discrete series representations of real rank one semisimple groups . . . . . . . . . . 299

Jeffrey Carroll, Some undecidability results for lattices in recursion theory

Gerald Howard Cliff and Alfred Rheinhold Weiss, Crossed product and

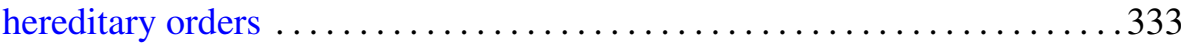

Ralph Cohen, Realizing transfer maps for ramified coverings . . . . . . . . 347

Ronald James Evans, Hermite character sums . .................. 357

C. L. Frenzen and Roderick Sue-Chuen Wong, Asymptotic expansions of the Lebesgue constants for Jacobi series . . . . . . . . . . . . . . . . 391

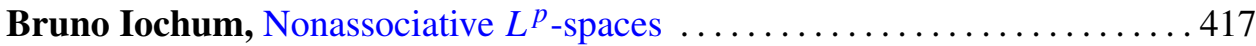

John McDonald, Unimodular approximation in function algebras ....... 435

John Robert Quine, Jr., Ramification and unintegrated value distribution . . 441

Marc Raphael, Commutants of quasisimilar subnormal operators ........ 449

Parameswaran Sankaran and Peter Zvengrowski, On stable

parallelizability of flag manifolds

Helga Schirmer, A relative Nielsen number

Barry Simon, Schrödinger semigroups on the scale of Sobolev spaces . . . . . 475

Viakalathur Shankar Sunder, Stochastic integration in Fock space

Jan de Vries, A note on the $G$-space version of Glicksberg's theorem 